\title{
Conceptualisation de l'accompagnement des maitres de stage et analyse de ses effets chez les stagiaires
}

Conceptualisation of the support provided by training supervisors and analysis

of its effects on trainees

Charlotte Dejaegher, Fanny Watelet, Yves Depluvrez, Stéphanie Noël et Patricia Schillings

\section{OpenEdition}

Journals

Édition électronique

URL : http://journals.openedition.org/activites/4183

DOI : 10.4000/activites.4183

ISSN : $1765-2723$

Éditeur

ARPACT - Association Recherches et Pratiques sur les ACTivités

Référence électronique

Charlotte Dejaegher, Fanny Watelet, Yves Depluvrez, Stéphanie Noël et Patricia Schillings, « Conceptualisation de l'accompagnement des maitres de stage et analyse de ses effets chez les stagiaires », Activités [En ligne], 16-1 | 2019, mis en ligne le 15 avril 2019, consulté le 09 août 2019 URL : http://journals.openedition.org/activites/4183 ; DOI : 10.4000/activites.4183

Ce document a été généré automatiquement le 9 août 2019

\section{c) (i) (8) $\Theta$}

Activités est mis à disposition selon les termes de la licence Creative Commons Attribution - Pas d'Utilisation Commerciale - Pas de Modification 4.0 International. 


\title{
Conceptualisation de
} l'accompagnement des maitres de stage et analyse de ses effets chez les stagiaires

\author{
Conceptualisation of the support provided by training supervisors and analysis \\ of its effects on trainees
}

Charlotte Dejaegher, Fanny Watelet, Yves Depluvrez, Stéphanie Noël et Patricia Schillings

\section{NOTE DE L'ÉDITEUR}

Article soumis le 14 mai 2018, accepté le 31 octobre 2018

\section{Introduction}

1 L'analyse de l'accompagnement des novices suscite un intérêt croissant dans une grande variété de professions avec pour cadre l'ergonomie et la didactique professionnelle (Filliettaz, Rémery, \& Trébert, 2014). En Fédération Wallonie-Bruxelles, l'accompagnement de futurs enseignants par des maitres de stage n'est encore que peu documenté et surtout est régi par peu de prescrits (Van Nieuwenhoven, \& Colognesi, 2015). En effet, les attendus en termes de gestes et de postures sont flous et aucune formation n'est obligatoire pour occuper la fonction de maitre de stage. Sur la base de ce constat, il semble légitime de se questionner sur la manière dont les enseignants endossent concrètement cette fonction parallèlement à leur activité d'enseignement. Sur quels éléments basent-ils leurs pratiques d'accompagnement des stagiaires ? Comment agissent-ils ? Observe-t-on des pratiques similaires chez des maitres de stage différents? Se pencher sur le modèle opératif (Pastré, 2011) des maitres de stage afin d'identifier ce 
qui oriente leur activité professionnelle conduit à formuler des hypothèses de réponses à ces différentes questions. En effet, dégager des organisateurs de l'action permet de cibler la manière dont des instituteurs primaires endossent leur rôle de maitres de stage et ce qui guide leur activité.

Dans un second temps, et pour tenter de percevoir les effets de l'activité des maitres de stage, les stagiaires ont été interrogés afin de cerner leur vision de l'accompagnement reçu et des bénéfices qu'ils en retirent. On peut en effet penser que les futurs enseignants ne vivent pas des expériences de stage similaires. Dans cette optique, le développement des compétences et de l'identité professionnelle durant la période de stage, est fortement influencé par le maitre de stage qui les accompagne, ce professionnel que Dubar (2000) considère comme un potentiel autrui significatif.

Cet article vise donc à étudier la manière dont ces maitres de stage comblent le manque de prescrits. Les analyses visent à (1) identifier les concepts qui organisent leur action durant les débriefings à chaud, (2) pointer les écarts éventuels entre les buts qu'ils assignent aux débriefings et leur fonctionnement effectif en situation et (3) analyser les effets de leur accompagnement perçus par les stagiaires.

\section{Le rôle de maitre de stage en Fédération Wallonie- Bruxelles de Belgique}

4 Le maitre de stage intervient à une étape où le futur enseignant s'essaye à son futur métier dans une situation professionnelle réelle. La tâche étant ardue, un accompagnement du novice est nécessaire. De Backer (2004) met par ailleurs en évidence une condition favorable à une pratique efficace du tutorat; selon lui, un préalable indispensable à l'accompagnement du formé est en effet que le tutorat soit organisé, c'est-à-dire qu'il y ait une reconnaissance de l'activité tutorale qui ne s'inscrit pas seulement dans la relation duale, mais qui soit instituée.

\subsection{Les prescrits légaux relatifs à la formation initiale ou continue}

5 En Fédération Wallonie-Bruxelles, les enseignants ne sont contraints à aucune formation pour devenir maitres de stage. L'article 20 du décret définissant la formation initiale des instituteurs et des régents (1997, p. 6), signale que « les maitres de stage qui accueillent dans leur classe des étudiants de $2^{\mathrm{e}}$ et $3^{\mathrm{e}}$ année sont agréés par les autorités de la haute école dans le cadre de l'accord de collaboration défini à l'article 23 du présent décret. Elles peuvent leur assurer une formation complémentaire ». Hormis l'accord des hautes écoles, il n'est fait mention d'aucun prérequis ou critère rogatoire tel que l'obligation d'avoir suivi une formation initiale particulière.

6 Par ailleurs, en Belgique francophone, aucune formation continue n'est requise pour exercer cette fonction. En outre, bien que certaines écoles organisent parfois des journées de rencontre autour de la définition du rôle et que l'Institut de Formation en Cours de Carrière propose une formation intitulée "Accueillir et accompagner de futurs instituteurs(-trices) en tant que maitre de stage : une fonction spécifique», ce genre de formations est peu répandu et peu plébiscité par les enseignants. Van Nieuwenhoven et Colognesi $(2013,2015)$ mettent par ailleurs en évidence que l'absence de balises explicites 
structurant les attendus relatifs au rôle des maitres de stage ne favorise pas une pratique effective d'accompagnement cohérente avec les acteurs de l'institut de formation.

\subsection{Les prescrits légaux relatifs au rôle du maitre de stage}

7 Nous l'avons montré, les prescrits en termes de formation, qu'elle soit initiale ou continuée, sont pour ainsi dire inexistants. Par conséquent, tout professionnel désireux d'endosser ce rôle de maitre de stage peut occuper cette fonction. Mais quels sont les attendus décrétaux quant à celle-ci ?

La fonction de maitre de stage peut être définie comme " un accompagnement par des professionnels expérimentés d'apprenants en situation de travail " (Boru, 1996, p. 99). Barnier (2001) indique que le tutorat prend place lors d'un moment où le novice s'essaye à son futur métier dans une situation professionnelle réelle. Selon l'article $3 \mathrm{du}$ décret définissant la formation initiale des instituteurs et des régents (2001, p. 2), les maitres de stage, en Fédération Wallonie-Bruxelles, «fournissent un accompagnement méthodologique aux stagiaires en formation et coopèrent avec les enseignants de la haute école dans l'évaluation formative des stagiaires qu'ils accompagnent». Ce terme d'accompagnement, n'étant à aucun endroit défini dans les textes légaux, c'est par conséquent son acception courante qui fait office de principale prescription, sans plus de précision.

9 Ainsi, le maitre de stage serait un guide dont la tâche est de permettre au stagiaire de "se distancier de la pratique de la classe en parlant de ce qui a été fait, de la manière dont cela a été fait, des raisons qu'il y a d'agir ainsi, etc.: une manière de développer son " aptitude à analyser sa pratique professionnelle et le contexte dans lequel il l'exerce " (B.O. no 22, du 25.05.97). Cette prise de recul vise à assurer au travail mené en stage un caractère formatif et, par conséquent, à favoriser chez l'étudiant un processus de développement de compétences professionnelles spécifiques (Barnier, 2001).

\subsection{Le concept d'accompagnement au regard de la littérature de recherche}

Le rôle des maitres de stage n'étant pas clairement défini dans nos prescrits en Fédération Wallonie-Bruxelles, nous présenterons brièvement dans ce point quelques balises théoriques quant à ce rôle et plus particulièrement la tâche d'accompagnement, son but, les postures qui peuvent y être endossées et l'efficacité plus ou moins relative de ces dernières en fonction des situations.

11 Chaque enseignant endosse son rôle de maitre de stage à sa manière, en se basant sur ses propres expériences dans le domaine, en y assimilant sa touche personnelle, son « style ». Selon Beauvais (2008) il n'y a pas de posture parfaite d'accompagnement. Celle-ci émergerait de la situation et de la relation avec le formé. Cependant, certaines balises sont nécessaires : toutes les postures ne sont pas adéquates dans toutes les situations. Par ailleurs, la relation tutorale est également fortement marquée d'un aspect socio-affectif. Ceci implique «que les échanges y sont vivants, que les propos concernant les exigences de la tâche sont approfondis, mais qu'en même temps ils sont personnalisés et gardent une certaine intimité » (Barnier, 2001, p. 216). Ces éléments mettent en évidence la portée de la qualité du climat, de l'environnement et de la relation tutorale et surtout l'influence déterminante de la facette « humaine » de ces métiers. L'importance de la collaboration 
entre maitre de stage et stagiaire ne doit toutefois pas éclipser le lieu de formation de l'étudiant, qui va, via ses prescriptions et la représentation des professeurs lors des visites de stage organisées, ici par la haute école, influencer inévitablement le fonctionnement du duo « maitre de stage-stagiaire».

\subsubsection{Un rôle multifinalisé}

12 Si accompagner revient à viser le développement de l'autre pour l'amener à complexifier son répertoire de manière à multiplier ses options dans l'environnement au sein duquel il évolue et sur lequel il agit (Beauvais, 2008), le rôle du maitre de stage ne se limite pas à cet accompagnement: il a aussi un rôle d'évaluateur du développement du stagiaire. En effet, celui-ci fait partie intégrante de l'accompagnement, «il ne peut y avoir de "bon" accompagnement sans "bon" jugement » (ibid., p. 123), ce qui peut être source de malaise pour le maitre de stage. En effet, "peut-on, et comment, passer de la posture de formateur, d'accompagnateur, voire d'accompagnant, à celle d'évaluateur, de contrôleur, de certificateur? [...] Le passage de la posture de celui qui forme, qui aide, qui accompagne, à celle de celui qui juge, qui estime, qui mesure, qui se prononce sur la valeur de l'Autre et/ou de ce qu'il produit, qui valide et sanctionne semblerait générateur d'un certain malaise et, pour peu que celui qui le ressent ne s'empresse pas de le refouler, d'un véritable cas de conscience» (ibid., p. 123). En Fédération Wallonie-Bruxelles, la plupart des hautes écoles pédagogiques accordent une attention plus ou moins importante à l'évaluation réalisée par le maitre de stage à l'issue de ce dernier. À la pauvreté de la définition du rôle s'ajoute donc une ambivalence entre deux fonctions à assurer, éventuellement source de tension identitaire (Bajoit, 2003). Cette tension a notamment été mise en évidence dans une étude menée par Maes, Colognesi et Van Nieuwenhoven (2018) auprès de 18 formateurs de haute école lors de l'entretien qui suit leur visite de stage.

\subsubsection{Accompagnement et expertise, comment les conjuguer?}

13 Les maitres de stage ont chacun leur propre expérience d'enseignement qui peut être plus ou moins importante selon leur ancienneté. De cette expérience peut naitre une expertise qui leur permet d'être efficaces dans une grande variété de situations. Dans le cadre de son rôle de maitre de stage, comment l'enseignant peut-il mobiliser cette expertise ? Bien que datée, la distinction établie par Schön (1988) entre les styles d'accompagnement « expert » et " praticien réflexif » demeure un cadre d'analyse éclairant à cet égard. Alors que l'expert se considère, par sa compétence, légitime et supérieur à la personne qu'il accompagne, le mentor peut être défini, selon Forest et Lamarre (2009), comme une personne qui conseille de manière avisée dans le but de guider quelqu'un. Ces auteurs précisent qu'il peut "exploiter son expérience pour contribuer à faire acquérir au stagiaire des comportements professionnels » (ibid., p. 8). Schön (1988) considère ainsi la relation expert/novice comme asymétrique lorsque le premier fournit au second des solutions, dont il peut même parfois douter, le but étant surtout de s'assurer à tout prix qu'il possède le savoir. Cette posture caricaturale renvoie néanmoins à plusieurs attitudes pouvant être adoptées par l'accompagnateur, mises en lumière par Dugal (2009) dans son modèle relatif aux positionnements des tuteurs lors des entretiens de débriefing; à savoir ici l'évaluation, l'interprétation et la décision. Le praticien réflexif ou compagnon réflexif, qualifié également de facilitateur (Schön, 1988), perçoit quant à lui un potentiel chez la 
personne qu'il accompagne. Il cherche donc à faire accepter ce qu'il sait en établissant une relation sincère et en agissant librement. Cette posture est de fait à associer à un accompagnateur qui laisse le stagiaire s'exprimer, qui est à l'écoute et qui surtout l'invite à problématiser, au sens entendu par Beckers, Biemar, Boucenna, Charlier, François et Leroy (2013). Pour ce faire, il adoptera davantage des attitudes d'investigation, de compréhension et parfois de soutien (Dugal, 2009). Ainsi, le facilitateur accompagne le futur enseignant dans la construction de solutions quand l'expert les lui fournit directement.

Dans la même optique, Portelance (2008) indique que le maitre de stage devrait fonctionner comme un guide plutôt qu'un modèle et pouvoir se détacher de sa propre identité enseignante pour accepter celle du stagiaire et l'aider à la développer évitant ainsi qu'il ne devienne une copie conforme de lui-même (Altet, Paquay, \& Perrenoud, 2002). Deux écueils sont à éviter : le premier consiste à s'imposer comme modèle unique et référent, le second est de ne rien proposer et de laisser le stagiaire cheminer seul. Mais quelle(s) posture(s) adopter pour devenir ce guide décrit par Portelance (2008), ce praticien réflexif (Schön, 1988)?

\subsubsection{Les postures du maitre de stage lors des débriefings post-observation}

L'empan de l'accompagnement en tant que maitre de stage est très large et couvre une série de tâches réparties sur plusieurs phases temporelles: la préparation du stage, l'organisation de l'accueil du stagiaire, la supervision du stage et l'organisation de la fin du stage (Pirard, François, \& Noël, 2015). Dans le cadre de notre recherche, nous nous centrerons sur la supervision du stage et plus particulièrement sur la tâche de débriefing avec le stagiaire.

Jorro (2016) identifie cinq intentions d'agir spécifiques de l'accompagnement professionnel d'enseignants débutants lors des débriefings. Selon cette auteure, les formateurs conscientisent des intentions qui leur «tiennent à cœur» et qu'ils convertissent en attitude. À partir de l'analyse de pratiques de 13 formateurs chevronnés en situation d'entretien post-observation de classe, Jorro (op. cit.) infère cinq postures caractéristiques de cette phase particulière de l'accompagnement. La posture de pisteur consiste à poser le cadre de l'entretien, à organiser les places respectives de chacun et à aménager l'espace de travail. La posture de contradicteur renvoie à l'écoute active et constructive, à la prise en compte des non-dits et des significations cachées. La posture de traducteur vise à rendre transparents des points non identifiés par le stagiaire ou à déconstruire des représentations erronées. La posture de médiateur vise à créer du lien que ce soit avec des ressources didactiques ou en mobilisant des gestes de conseil. Enfin, la posture de régulateur, qui peut valoriser les réussites de l'activité, renvoie le stagiaire à une démarche d'autoévaluation.

\section{La didactique professionnelle comme cadre d'analyse}

Nous avons montré que Jorro (2016) identifiait, lors des moments de débriefings postobservation, des intentions d'action qui semblent porteuses. Plus que les intentions d'action des maitres de stage, il parait intéressant de mieux comprendre le lien entretenu entre ces intentions et leur concrétisation sur le terrain. Ainsi, nous essayerons de déterminer si les intentions d'agir de nos maitres de stage sont en adéquation avec leur 
activité effective lors du moment de débriefing post-observation. Pour investiguer cette question, le cadre d'analyse proposé par la didactique professionnelle nous semble prometteur.

La didactique professionnelle repose sur l'analyse de l'activité et l'examen des connaissances qui sont mises en acte par les professionnels. Cette approche nous a conduits à appréhender la manière dont les maitres de stage agissent en situation. Par conséquent, cette étude cible l'observation de ce qu'ils mettent en œuvre lors des débriefings avec les stagiaires, comment ils le font et ce qui guide leur activité. Ce cadre d'analyse souligne également l'intérêt de décrire la manière dont le professionnel met en œuvre les différents niveaux de tâches décrits par Leplat (2011) : "tâche prescrite", " tâche représentée », « tâche effective » et « tâche réalisée ».

\subsection{Le professionnel dans une tâche discrétionnaire : quatre niveaux de tâches décrites par Leplat (2011)}

La tâche prescrite est définie en termes de but à atteindre et de conditions dans lesquelles la réaliser (Veyrac, 1998). Le degré de précision de la description de la tâche prescrite peut différer. Ainsi, en comparaison avec les métiers du secteur industriel où cette description est très procéduralisée, les métiers de service rencontrent notamment une plus grande part d'imprévisibilité et conduisent à une plus grande autonomie du travailleur. C'est notamment le cas de la tâche d'accompagnement de stagiaires qui peut être qualifiée de discrétionnaire: «il y a obligation de résultats sans certitude de moyens » (Pastré, 2007, p. 87). En effet, les maitres de stage doivent sans conteste mener un important travail d'élaboration et d'interprétation, qu'il s'agisse de situations de diagnostic ou de prise de décision. Ce travail d'interprétation, amène le sujet à construire " la tâche redéfinie ", c'est-à-dire la représentation que s'est construite le sujet à propos de la tâche et la manière dont il doit la réaliser (Leplat, 2011). Cette représentation, que Weill-Fassina, Rabardel et Dubois (1993, p.7) définissent comme un «réseau de propriétés, de concepts, de savoirs, de savoir-faire, de croyances, de sensations éprouvées construites, sélectionnées au cours de l'histoire du sujet, à partir de sa formation, de son expérience, et des besoins de l'action ", est par définition propre à chaque individu. La tâche effective correspond quant à elle aux actions réellement mises en œuvre par le professionnel; elle renvoie aux buts et aux conditions d'exécution effectivement pris en considération par le professionnel lors de son activité. Enfin, la tâche réalisée correspond au résultat observable de l'activité du sujet. Celle-ci peut être décrite à l'aide d'une observation « armée » (enregistrement vidéo, grille d'observation) de l'activité du sujet.

C'est principalement l'analyse de la tâche effective lors des débriefings de nos deux sujets qui est visée dans le cadre de cet article.

\subsection{La conceptualisation dans l'action}

21 Trois concepts sont à la base de la conceptualisation dans l'action en didactique professionnelle : les concepts pragmatiques, la structure conceptuelle d'une situation et le modèle opératif. Pastré (2011, p. 172) définit le concept pragmatique comme «un concept construit dans l'action et servant à guider l'action, associé à un ou plusieurs indicateurs ». Sa première propriété est sa fonction : il organise l'action du professionnel en établissant un diagnostic de situation. Il est par conséquent toujours relié à des 
indicateurs, inhérents à l'action et donc éloignés de la théorie. Dans la pratique, le concept pragmatique pourrait même presque disparaitre, laissant place à l'indicateur de situation sur lequel le professionnel porte son attention tout entière. Sa seconde propriété est son origine, il émerge de l'action et non de définitions. Il dispose malgré tout d'une dimension sociale, c'est là sa troisième propriété. En effet, les concepts pragmatiques semblent être transmis des professionnels aux novices, mais de façon particulière: les professionnels en parlent, montrent, effectuent des gestes, mais ne définissent généralement pas le concept pragmatique. En effet, la quatrième propriété du concept pragmatique est que personne ne le définit explicitement, mais que chacun sait globalement de quoi il est question. Notons également que les concepts pragmatiques ont une double face: la première, la face objective, tournée vers l'action en temps réel et la seconde, la face subjective, orientée vers l'organisation du réseau des représentations du sujet (Vidal-Gomel \& Rogalski, 2007).

La didactique professionnelle envisage le professionnel comme "sujet capable" (Vinatier, 2009). Il est en effet essentiel que la réflexion et la conceptualisation viennent de lui pour qu'il puisse s'en resservir par la suite pour orienter son activité. Cependant, différents obstacles à la compréhension de son niveau de conceptualisation dans l'action par le chercheur peuvent être rencontrés. Premièrement, il y a un écart entre ce que le professionnel pense faire, ou désire faire, et ce qu'il fait effectivement. Il y a donc une différence entre ce que Leplat (2011) définit comme la tâche redéfinie et la tâche effective qu'il convient de prendre en compte dans les analyses. Ensuite, le sujet n'accède pas directement à une compréhension des processus interactionnels mis en œuvre en situation (Pastré, 2005). Enfin, il est contre-intuitif de verbaliser ses actions. Le fait que l'action soit en partie implicite et qu'il soit nécessaire de se la remémorer pour pouvoir en parler contribue à accroitre la difficulté de l'activité d'analyse. Recourir à des techniques d'entretien telles que celle proposée par Vermersch (1994) ou Beckers (2012) permet, grâce à l'explicitation par le professionnel de son activité, d'accéder à une meilleure compréhension des concepts qui guident leur action. Ces entretiens ne se résument bien évidemment pas à une simple mise en mots ; pour Vinatier (2009), bien que l'enseignant puisse décrire son activité, lorsqu'il passera à l'analyse, sa subjectivité entrera en jeu. C'est pourquoi le travail de l'accompagnateur lors de l'entretien conditionne la qualité des informations qui pourront y être collectées. Dans le point suivant, nous décrirons les étapes et critères retenus par Vermersch pour conduire un entretien d'explicitation de qualité.

\section{Méthodologie}

\subsection{Participants et recueil de données}

Dans le cadre de cette recherche, quatre maitres de stage (MS) ont été suivis. Cet article vise l'analyse de l'activité d'accompagnement des stagiaires chez deux de ces sujets. 
Tableau 1 : Présentation des sujets.

Table 1: Description of the participants

\begin{tabular}{|c|c|c|c|c|c|c|}
\hline$M S$ & Sexe & Stagiaire & $\begin{array}{c}\text { Niveau scolaire } d u \\
\text { stagiaire }\end{array}$ & Sexe & $\begin{array}{l}\text { Formation } \\
\quad M S\end{array}$ & Expérience $\mathrm{MS}$ \\
\hline MS 1 & $\mathrm{~F}$ & Jérémy & $\begin{array}{l}2^{\mathrm{e}} \text { année de bachelier } \\
\text { instituteur primaire }\end{array}$ & $\mathrm{M}$ & Non & $\begin{array}{l}\text { une dizaine de } \\
\text { stagiaires }\end{array}$ \\
\hline MS 2 & F & Joseph & $\begin{array}{l}3^{\mathrm{e}} \text { année de bachelier } \\
\text { instituteur primaire }\end{array}$ & M & Oui & $\begin{array}{l}\text { une dizaine de } \\
\text { stagiaires }\end{array}$ \\
\hline
\end{tabular}

Le recueil de données comporte cinq étapes étalées sur une période de trois mois et schématisées dans la figure 1. Une rencontre préalable à la récolte de données ciblée dans cette étude a permis d'établir un premier contact, de réexpliquer le projet et de mener le premier entretien semi-directif concernant leur parcours et leurs représentations sur le rôle de maitre de stage (étape 1). Les trois étapes suivantes de recueil de données ont été reproduites à deux moments du stage : observation par la chercheure de deux leçons données par le stagiaire y compris des éventuelles interventions du maitre de stage (étape 2), observation du débriefing ${ }^{1}$ maitre de stage - stagiaire (étape 3), entretien d'explicitation avec le maitre de stage (étape 4). Enfin, un entretien semi-directif était mené avec le stagiaire afin de revenir sur l'accompagnement dont il avait bénéficié (étape 5).

Figure 1 : Étapes de récolte de données.

Figure 1: Data collection sequence

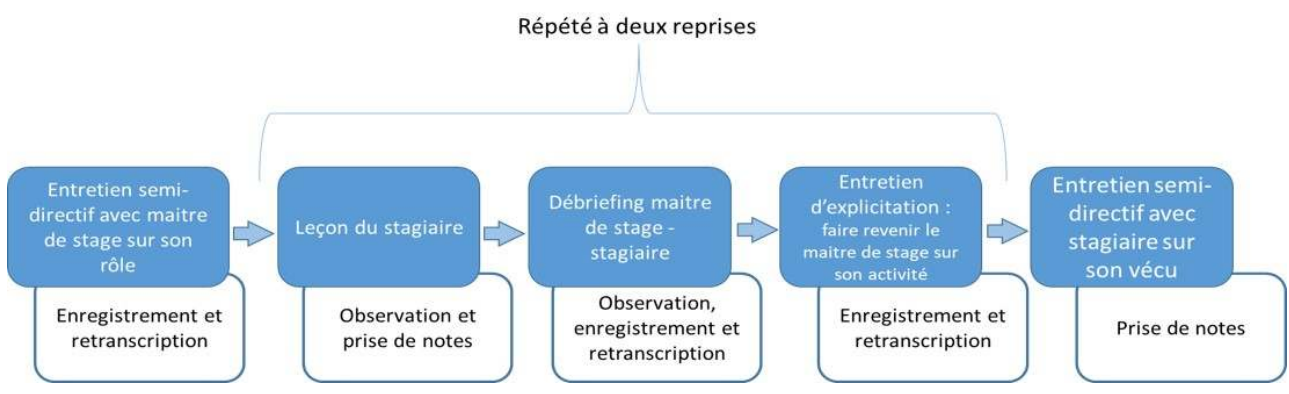

Lors de la quatrième étape, nous mènerons des entretiens en recourant à des techniques d'explicitation. Au début des entretiens, le maitre de stage avait au départ l'opportunité de revenir sur un élément de son action à sa convenance. L'occasion lui était donnée de verbaliser son vécu, et plus particulièrement un événement qui lui faisait sens. À partir de ce premier échange, le professionnel était amené à se concentrer davantage sur la description du vécu de l'action. «Que visez-vous quand vous faites ça? ; « Quel est alors votre but?»; «En quoi consiste cette action pour vous?»; «Que va-t-elle amener?», sont différents types de questions de relance utilisés par le chercheur durant l'entretien pour rediriger la verbalisation sur un vécu d'action et éviter que le professionnel s'oriente trop vers les satellites de l'action (Vermersch, 2004).

\subsection{Traitement des données : élaboration du modèle opératif}

Sur la base des transcriptions des entretiens d'explicitation (étape 4), lorsque les professionnels décrivaient les éléments pris en compte pour orienter leur action (les indicateurs et règles d'action) et suite à une analyse qualitative proche de la méthode proposée par l'Écuyer (1987), différentes composantes du modèle opératif des maitres de 
stage ont pu être inférées. Le modèle opératif de chacun des maitres de stage sera présenté dans une figure faisant apparaitre les indicateurs, les variables et les concepts organisateurs de leur action. Par définition, les modèles opératifs présentés seront donc adaptés à chacun des deux sujets, notamment au niveau du nombre de concepts organisateurs présentés, du nombre de variables, ou encore du nombre d'indicateurs et de leur agencement. Les indicateurs pris en compte dans le déroulement du débriefing sont liés à l'environnement, donc, dans ce cas, au stagiaire, aux élèves, au climat de classe, etc. Ils ont été regroupés en variables qui rendront compte, quant à elles, d'un concept organisateur. Précisons qu'afin d'augmenter la fidélité des résultats, plusieurs chercheurs ont fait l'analyse afin d'avoir une fidélité « inter-chercheur » de $80 \%$.

\section{Résultats}

\subsection{Le modèle opératif des maitres de stage}

27 L'analyse des propos tenus par le professionnel à propos de son action lors des entretiens d'explicitation vise à identifier des éléments susceptibles de fonctionner comme des organisateurs de son action. Des concepts pragmatiques et leur(s) indicateur(s) regroupés en variables ont ainsi été inférés et modélisés. Pour chaque concept pragmatique, entre deux et trois indicateurs ont été définis et regroupés en variables selon l'objet sur lequel ils portaient.

\subsubsection{Modèle opératif de la maitre de stage 1}

L'analyse des entretiens d'explicitation menés avec la MS 1 a permis d'élaborer un modèle opératif susceptible d'éclairer l'activité d'accompagnement mise en œuvre par cet enseignant.

Figure 2 : Modèle opératif de la maitre de stage 1.

Figure 2: Supervisor 1's operative model

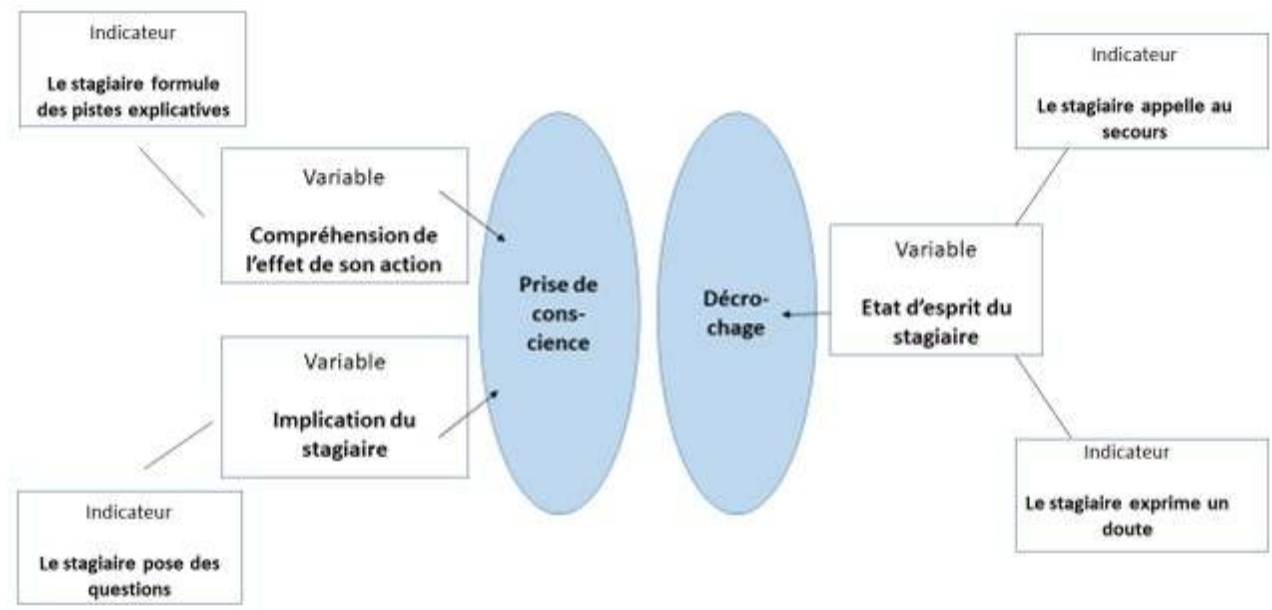




\section{un modèle opératif
cette enseignante.}

Figure 3 : Modèle opératif de la maitre de stage 2.

Figure 3: Supervisor 2's operative model

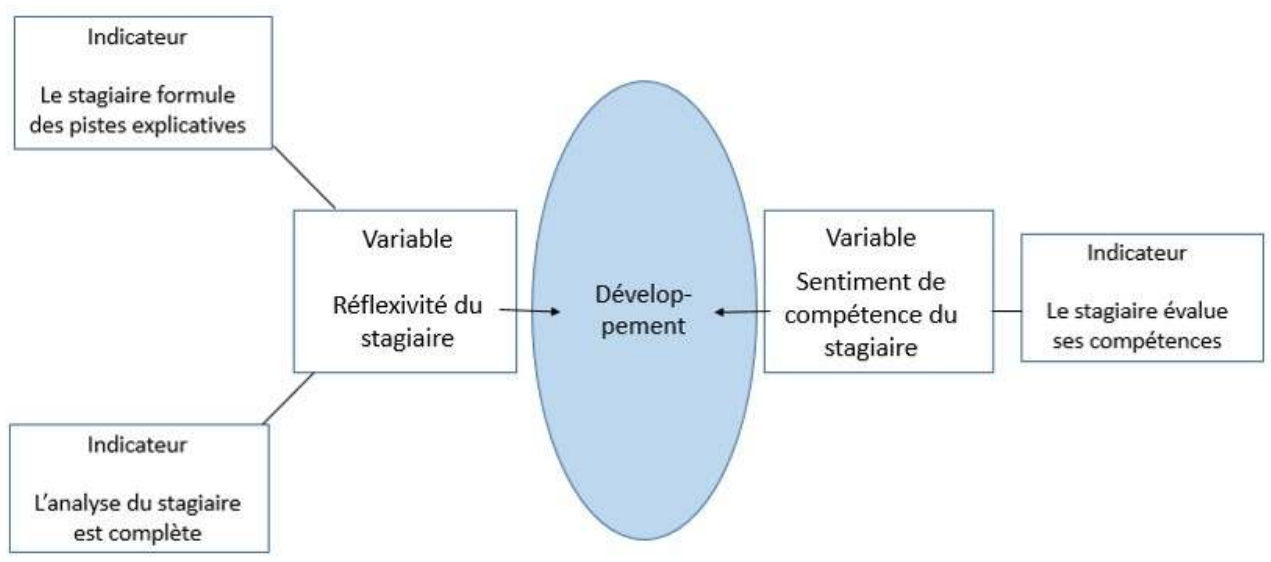

concept pragmatique central apparait dans le modèle opératif de la seconde maitre de stage : le développement professionnel du stagiaire, à long terme. Ce concept est lié à une première variable : la réflexivité du stagiaire. Pour déterminer ce degré de réflexivité, la maitre de stage se base sur la prise en compte de deux indicateurs: le fait que le stagiaire s'autoanalyse et que son analyse soit complète. Le concept de " développement » est également en lien avec une deuxième variable fonctionnelle: le sentiment de 
compétence du stagiaire. La MS prend comme indicateur la verbalisation par le stagiaire de son sentiment de compétence.

\subsection{Mise en lien du modèle opératif, de la verbalisation sur l'action et de l'action effective}

Dans cette partie, chacune des variables fonctionnelles identifiées dans les modèles opératifs des maitres de stage est mise en lien avec l'activité de débriefing. Le discours sur l'activité, recueilli à l'étape 4 du recueil de données, est confronté à l'activité de débriefing observée à l'étape 3 .

\subsubsection{Chez la maitre de stage 1}

Parmi les concepts qui organisent l'activité de tutelle de la maitre de stage, deux concepts centraux ont été mis en lumière : la prise de conscience et le décrochage.

L'action de l'enseignante semble guidée par l'idée selon laquelle le stagiaire doit prendre conscience d'une série d'éléments de son activité qu'elle juge cruciaux. Lorsqu'elle verbalise à propos des éléments qui guident son action, MS 1 aborde le fait que le stagiaire ne comprend pas toujours pourquoi ses activités n'ont pas bien fonctionné. C'est ce qui a été objectivé par des verbatim dans la première colonne du tableau 1. Cette objectivation a permis d'établir une première variable fonctionnelle « compréhension par le stagiaire de l'effet de son action » comme un des éléments guidant l'action de la maitre de stage. Le fait que le stagiaire ne semble pas toujours comprendre les effets de ses démarches ou de ses choix renseigne la maitre de stage sur son niveau de prise de conscience qui parait déficitaire et auquel elle va donc devoir remédier activement.

Liens entre les données du débriefing et celles de l'entretien d'explicitation de MS 1 pour la variable fonctionnelle "compréhension par le stagiaire de l'effet de son action" 
Tableau 2 : Liens entre les données du débriefing et celles de l'entretien d'explicitation de MS 1 pour la variable fonctionnelle « compréhension par le stagiaire de l'effet de son action ». Table 2: Links between data from the debriefing and those from the clarificatory interview of MS 1 for the functional variable "trainee's understanding of the effect of her action"

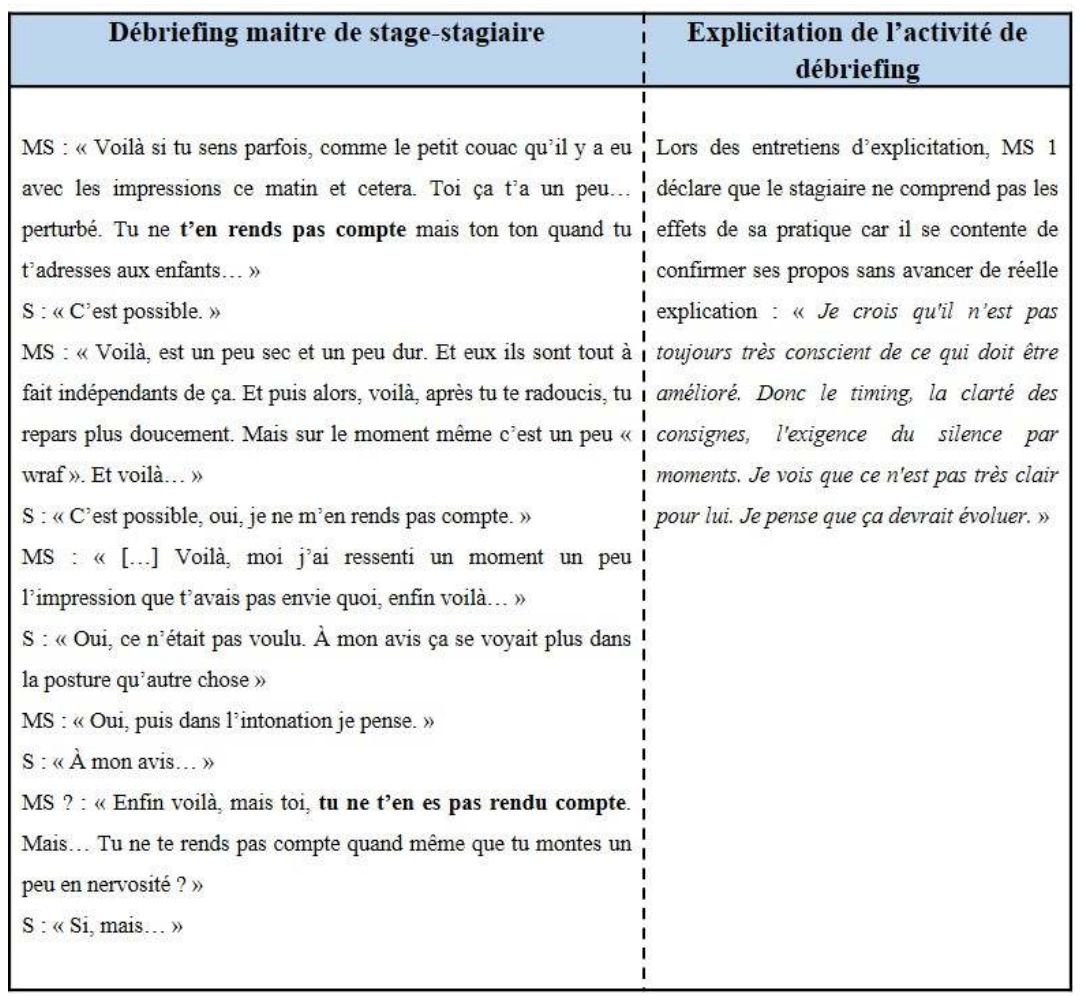

D'après Pastré (2011), l'action est guidée par des concepts pragmatiques bâtis sur des indicateurs. MS 1 semble remarquer que son stagiaire ne fait que confirmer ses propos et qu'il ne propose pas lui-même d'explication. Sur la base de cet indicateur, elle établit comme diagnostic de situation que le stagiaire éprouve des difficultés à comprendre les effets de ses actions. Son objectif étant qu'il en prenne conscience (concept pragmatique), elle en vient à parler beaucoup, à donner son avis et à peu se pencher sur celui du stagiaire. En se basant sur le manque de compréhension qu'elle perçoit chez lui, elle semble estimer que c'est à elle de lui expliquer. Ainsi, lors d'un débriefing, elle proposera d'emblée son analyse quant aux difficultés de certains enfants, cherchant à lui révéler «ce qu'il n'a pas vu». Lors de l'entretien d'explicitation, elle précisera les raisons qui l'ont poussée à pointer elle-même les élèves en difficulté et à proposer une piste explicative :

«Je les connais fort bien et je sais d'emblée quand on va faire un travail de groupe qui va être le profiteur et va décider de ne rien faire, qui va être le meneur, qui il faudra tempérer au niveau du bruit... ».

Dans l'action, un grand temps de parole est donc pris par la maitre de stage qui fournit un retour au stagiaire sur ses activités d'enseignement. Elle donne son avis avec beaucoup de conviction et semble sûre de ce qu'elle avance. Les interventions prises en exemple dans les verbatim repris au tableau 1 relèvent de la posture d'expert telle que décrite par Schön (1988). Par moments, la maitre de stage questionne brièvement le stagiaire afin d'obtenir son ressenti, son idée sur la question. Il semblerait qu'il s'agisse plutôt ici de l'amener à confirmer son propos (« Non?»; «Tu vois?»). Cela rejoint à nouveau l'idée 
d'un accompagnateur « expert » qui met en avant sa lecture de la situation et qui adopte une posture d'interprétation telle que définie par Dugal (2009).

Néanmoins, lors de l'entretien préalable (étape 1), lorsque MS 1 est amenée à décrire son rôle, elle déclare envisager ces moments comme des discussions riches où autant le maitre de stage que le stagiaire prennent la parole pour analyser l'action et proposer des pistes.

Figure 4 : Verbatim de MS 1 illustrant sa représentation du débriefing.

Figure 4: MS 1's verbatim illustrating her vision of a debriefing

«Moi je pense que c'est un échange à deux niveaux. [...] Donc je pense que c'est un échange riche, dans les deux sens. [...] Parce que je pense que c'est à lui de voir ce qui a évolué et ce qui est encore à faire. Un débriefing ce n'est pas que moi qui dit "tac, tac, tac", c'est aussi lui qui doit être conscient de... Ça me parait évident. Enfin, ça me parait important qu'il y ait aussi une auto-analyse de... [...] Il faut qu'il ait quand même son mot à dire, c'est normal. "

41 La vision d'accompagnement de MS 1 telle qu'elle se l'est redéfinie relève de la problématisation et de l'initiation à l'analyse réflexive (Beckers et al., 2013). La démarche décrite évoque la posture du facilitateur qui suscite chez l'accompagné une capacité à analyser ses pratiques et à réfléchir à des moyens pour les améliorer (Schön, 1988). Toutefois, cette posture ne se reflète pas, ou très peu, dans son activité telle qu'observée par le chercheur et filmée (tâche réalisée) ni dans ce qu'elle peut en dire lors des entretiens d'explicitation (qui permettent d'inférer sur la tâche effective). En cause, la probable croyance chez elle que le stagiaire ne comprendrait pas son action si on ne l'y aide pas. Par conséquent, un décalage apparait entre la manière dont MS 1 envisage son rôle et celle dont elle l'endosse effectivement.

\section{Liens entre les données du débriefing et celles de l'entretien d'explicitation de MS 1 pour la variable fonctionnelle " implication du stagiaire "}

Le concept pragmatique concernant la " prise de conscience du stagiaire » repose sur une deuxième variable fonctionnelle intervenant lorsque MS 1 perçoit que le stagiaire s'implique dans son rôle, pose des questions et sollicite son point de vue dans un but de s'améliorer. Lors de l'entretien d'explicitation, MS 1 explique qu'elle considère ses questions comme une implication positive du stagiaire dans un processus de prise de conscience. Ainsi, dans l'action, pour aider le stagiaire à prendre conscience de certains éléments, MS 1 répond à ses questions en donnant son avis, en fournissant sa lecture de la situation, voire en donnant quelques conseils. 
Tableau 3 : Liens entre les données du débriefing et celles de l'entretien d'explicitation de MS 1 pour la variable fonctionnelle « implication du stagiaire ».

Table 3: Links between data from the debriefing and those from the clarificatory interview of MS 1 for the functional variable "trainee's commitment"

\begin{tabular}{|c|c|}
\hline $\begin{array}{l}\text { Débriefing maitre de stage - stagiaire } \\
\text { Tâche achevée }\end{array}$ & $\begin{array}{l}\text { Explicitation de l'activité de } \\
\text { débriefing } \\
\text { Tâche effective }\end{array}$ \\
\hline 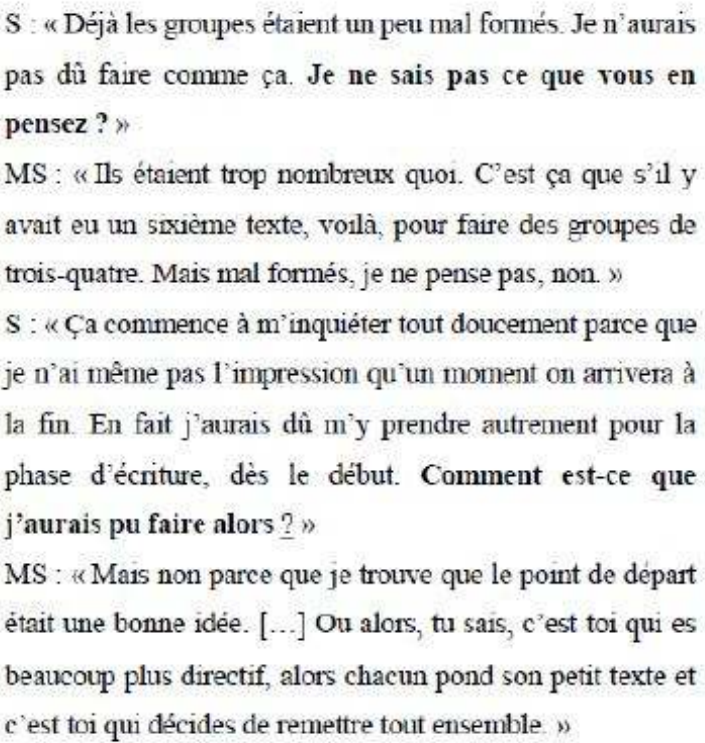 & $\begin{array}{l}\text { Lors d'un des entretiens d'explicitation, la } \\
\text { chercheure revient sur cet échange du } \\
\text { debriefing. La maitre de stage déclare: } \\
\text { «J'ai eu la chance de tomber sur un } \\
\text { stagiaire qui est très demandeur, qui pose } \\
\text { souvent des questions, qui veut souvent } \\
\text { savoir si tout a bien fonctionné, qu'est-ce } \\
\text { qu'il peut faire pour améliorer etc. } »\end{array}$ \\
\hline
\end{tabular}

43 À nouveau, l'extrait du débriefing présenté dans la colonne de gauche montre que la maitre de stage adopte une posture d'expert, une posture d'interprétation (Dugal, 2009) : elle répond aux questions du stagiaire en fournissant sa lecture de la situation. Et, nouvel élément, elle donne également quelques conseils en réponse aux interrogations du stagiaire et aux moments de l'activité s'étant moins bien déroulés. Dans son accompagnement effectif, MS 1 semblerait prendre appui sur la variable fonctionnelle «l'implication du stagiaire » dans le processus de prise de conscience (représentée dans le modèle opératif de MS 1 en figure 2) pour guider l'amélioration des pratiques pédagogiques de son stagiaire. Elle pose comme diagnostic qu'il a envie de s'améliorer et en réponse à cela, elle lui donne son avis et le conseille, comme illustré dans les échanges tirés de la retranscription des débriefings, et ce toujours dans le but que le stagiaire prenne conscience de l'effet produit par ses démarches d'enseignement. Elle adopte donc une posture de décision (Dugal, 2009). Cependant, comme constaté dans ses pratiques effectives, cela ne semble pas l'amener à inciter l'étudiant à analyser lui-même sa pratique. Le couplage de ces deux premières variables fonctionnelles en est peut-être la cause. En effet, MS 1 veut que le stagiaire prenne conscience de l'effet produit par ses pratiques. Elle observe pendant les débriefings que celui-ci ne le saisit pas, mais qu'il voudrait comprendre et qu'il désire s'améliorer. Par conséquent, elle analyse elle-même l'activité de ce dernier, elle lui fait part de sa lecture des faits à laquelle elle ajoute quelques conseils pour qu'il s'améliore à l'avenir. Cette façon d'accompagner s'apparente à une position d'expert qui, selon Musquer et Fabre (2011, p.135), fournit « une explication directe et "magistrale" des données et conditions souhaitées ". 


\section{Liens entre les données du débriefing et celles de l'entretien d'explicitation de MS 1 pour la variable fonctionnelle "état d'esprit du stagiaire"}

L'analyse des données nous a permis de mettre en évidence un deuxième concept organisant l'activité de la maitre de stage lors des débriefings: «le décrochage ». Ce concept est lié à la perception que la maitre de stage a de l'état d'esprit du stagiaire à certains moments de sa leçon ou lors des débriefings. Dans l'action du débriefing, quand MS 1 perçoit que le stagiaire perd confiance ou semble perdu, elle lui fournit des conseils précis pour le rassurer.

Tableau 4 : Liens entre les données du débriefing et celles de l'entretien d'explicitation de MS 1 pour la variable fonctionnelle " état d'esprit du stagiaire ".

Table 4: Links between data from the debriefing and those from the clarificatory interview of MS 1 for the functional variable "trainee's state of mind"

\begin{tabular}{|c|c|}
\hline $\begin{array}{c}\text { Débriefing maitre de stage - stagiaire } \\
\text { Tâche achevée }\end{array}$ & $\begin{array}{c}\text { Explicitation de l'activité de } \\
\text { débriefing } \\
\text { Tâche effective }\end{array}$ \\
\hline $\begin{array}{l}\text { MS : " [...] l'exercice du "math et sens" il est très bien fait. } \\
\text { Mais la fois passée.... " } \\
\text { S : « Oui ce n'était pas top. C'était des explications un petit } \\
\text { peu... " } \\
\text { MS : «Voilà, la consigne par rapport au circuit ça n'allait } \\
\text { pas. Donc il faudrait, demain, quand tu vas après aborder le } \\
\text { divisé par cinq, cinquante et cinq cents, que tu modifies un } \\
\text { petit peu ta manière de présenter l'exercice aux enfants. } \\
\text { Donc là je t'ai mis un petit peu [...] mais toutes les idées } \\
\text { qui me venaient par là. Je pense que tu peux commencer la } \\
\text { séquence demain par rappeler ce qui est déjả là. Donc voilà, } \\
\text { pas vraiment comme Jules César, mais avec Madame B. on } \\
\text { a vu fois dix, fois cent, fois mille ; divisé en dix, divisé en } \\
\text { cent, divisé en mille. Tu peux peut-être à l'avance déjà } \\
\text { écrire au tableau ça. Et puis alors, voilà, "on a déjà abordé } \\
\text { début de semaine, comment on multipliait par cinq et } \\
\text { cetera, maintenant on va passer aux divisions". Alors, on va } \\
\text { essayer de voir comment au départ de divisé par dix, divisé } \\
\text { par cent, on va pouvoir faire... » }\end{array}$ & $\begin{array}{l}\text { Lors de l'entretien d'explicitation, } \\
\text { MS } 1 \text { explique qu'elle était interpellée } \\
\text { par le stagiaire qui, selon elle, ne s'en } \\
\text { sortait pas pendant sa leçon et perdait } \\
\text { confiance en ses capacités. } \\
\text { "Fatalement avec une consigne mal } \\
\text { formulée, l'activité part dans tous les } \\
\text { sens. [...] Qu'est-ce qu'il était stressé. } \\
\text { Ça aussi il a fallu... L'air de rien, le } \\
\text { déstresser, le mettre en confiance.» } \\
\text { Elle précise les indicateurs qui la } \\
\text { conduisent à ce diagnostic: } \\
\text { « De temps en temps j'ai eu un petit } \\
\text { appel au secours, ou alors il me dit "je } \\
\text { ne sais pas que faire". [...] J'ai donné } \\
\text { des pistes pour expliquer clairement les } \\
\text { consignes. Donner un exemple au } \\
\text { tableau, faire venir un enfant pour } \\
\text { réexprimer la consigne... » }\end{array}$ \\
\hline
\end{tabular}

Éclairé par les données de l'entretien d'explicitation, cet extrait du débriefing stagiaire maitre de stage montre que la MS 1 met en place un cadrage serré, fournit des conseils très précis pour les leçons à venir, allant jusqu'à formuler un exemple de consignes que le stagiaire pourrait donner. MS 1 a clairement verbalisé deux indicateurs qui l'informaient sur l'état d'esprit du stagiaire : le fait que le stagiaire l'appelle au secours et le fait qu'il exprime ses doutes. La prise en compte de ces indicateurs et le désir de sortir le stagiaire de son état de « décrochage » ont engendré le fait qu'elle s'est mise à donner des conseils très précis pendant les débriefings. Ceux-ci avaient donc, semble-t-il, pour fonction d'éviter que le stagiaire soit perdu à nouveau. Le cadrage serré mis en place dans l'action est lui aussi caractéristique d'une posture d'expert. 


\subsubsection{Chez la maitre de stage 2}

L'activité de la maitre de stage lors des moments de débriefing est majoritairement organisée par un concept principal : le développement professionnel du stagiaire, dans une visée à long terme. Nous présenterons ci-après des extraits des débriefings et des entretiens d'explicitation pour chacune des variables liées à ce concept organisateur de l'action.

\section{Liens entre les données du débriefing et celles de l'entretien d'explicitation de MS 2 pour la variable fonctionnelle " réflexivité du stagiaire »}

Lors des entretiens d'explicitation, MS 2 explique qu'afin d'amener le stagiaire à plus de réflexivité, elle le fait écrire en fin de journée dans un carnet ce qu'il a pensé de ses leçons, ce qu'il aurait pu améliorer, etc. Elle lui demande de structurer ses écrits selon les catégories du rapport de stage (conception des dispositifs, mise en œuvre...). Lors des débriefings, elle commence toujours par lui demander ce qu'il a écrit. Elle approfondit ensuite la réflexion du stagiaire par des questions.

Tableau 5 : Liens entre les données du débriefing et celles de l'entretien d'explicitation de MS 2 pour la variable fonctionnelle " Réflexivité du stagiaire". Table 5: Links between data from the debriefing and those from the clarifying interview of MS 2 for the functional variable "trainee's reflexivity"

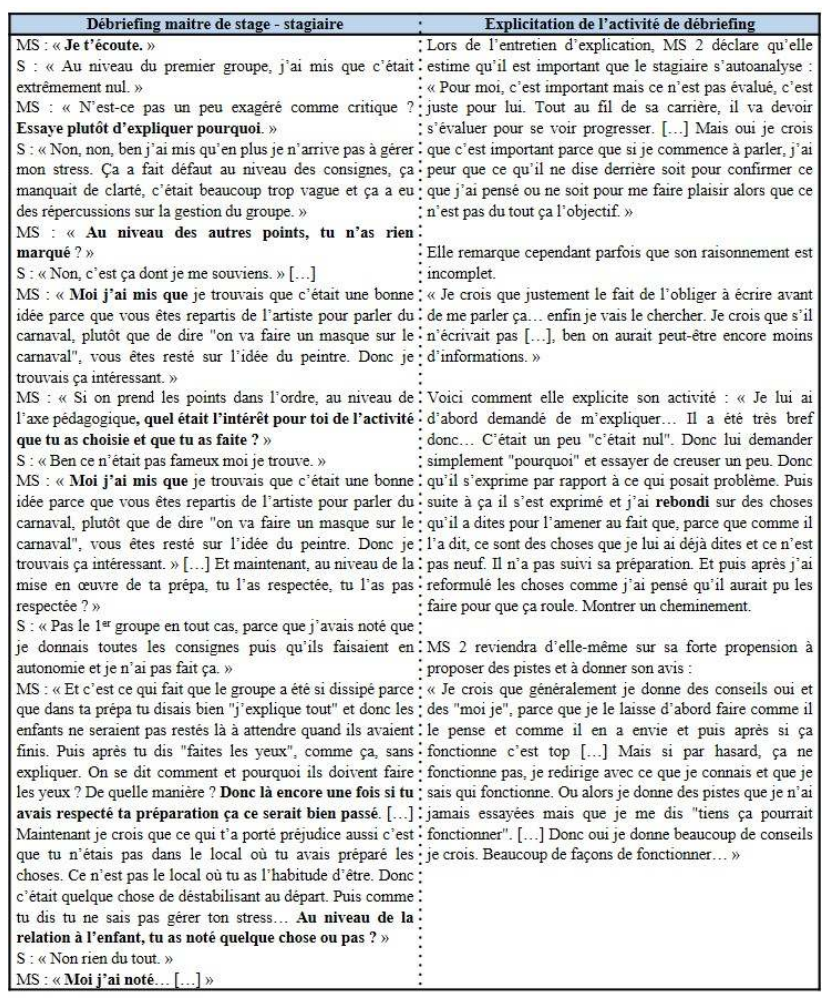

L'activité de la maitre de stage apparait organisée par le concept d'autonomie du stagiaire, dont une des variables majeures est la réflexivité. Lors de l'entretien d'explicitation, elle déclare à ce propos que le stagiaire est peu bavard et qu'elle doit " aller le chercher ». Elle se base sur la prise en compte de deux indicateurs pour établir son diagnostic en situation: le fait que le stagiaire formule des hypothèses en lien avec 
ses actions et la complétude du raisonnement proposé. En fonction de ces deux indicateurs, elle organise son action : elle lui pose des questions et tente de l'amener à poursuivre sa réflexion.

En début de débriefing, MS 2 agit comme un facilitateur et « s'évertue à déclencher et à soutenir des mécanismes autorégulateurs » (Teissier, 1992). En effet, en l'amenant à approfondir (« Essaye plutôt d'expliquer pourquoi »), elle soutient la réflexivité en plus de la déclencher. Si la posture de facilitateur est endossée par MS 2 comme porte d'entrée de l'entretien, la prévalence de l'indicateur «l'analyse du stagiaire est complète » semble néanmoins la conduire à un changement de posture assez rapide. En effet, dès que les réponses du stagiaire ne correspondent plus au niveau de ses indicateurs, elle change de posture : elle agit alors davantage comme un expert; l'incomplétude de l'analyse avancée par le stagiaire conduit MS 2 à lui faire part de ses observations et à intervenir dans l'analyse en s'appuyant sur son expérience professionnelle. Face aux actions, réactions et réponses du stagiaire, MS 2 semble amenée à agir à certains moments comme un expert qui fournit son avis et impose des solutions.

Lors de l'entretien d'explicitation, MS 2 revient sur ce changement de posture et l'explique par deux représentations. D'une part, elle considère que l'expérience professionnelle lui permet de percevoir des éléments que le stagiaire ne peut percevoir et qu'il est nécessaire de «lui donner d'autres pistes, telles que des petites améliorations, des petits détails que l'on a parfois acquis avec l'expérience et que l'on ne voit pas nécessairement la première fois qu'on fait quelque chose ». D'autre part, l'expérience professionnelle est, selon elle, transposable d'un individu à l'autre, et par conséquent ce qui est efficace pour elle l'est forcément pour le stagiaire. Ainsi, elle déclare :

«C'est peut-être gagner du temps aussi d'expliquer le chemin par lequel on est passé et qu'on a trouvé efficace ».

51 Lors de l'entretien préalable (étape 1), cette ambivalence entre les postures de facilitateur et d'expert transparait dans la manière dont MS 2 décrit son rôle pendant le débriefing.

Figure 5 : Verbatim de MS 2 illustrant sa représentation de la tâche de débriefing. Figure 5: MS2's verbatim illustrating her vision of a debriefing

« Justement, il [le maitre de stage] peut lui amener un miroir pour qu'il puisse se regarder et analyser entre guillemets. Il peut lui amener un regard, pour moi c'est vraiment le truc primordial. Mais il peut aussi lui partager son expérience, ses pratiques, ses idées ».

Cette ambivalence entre les postures pourrait également s'expliquer par une maitrise insuffisante des techniques d'entretien à visée réflexive lui permettant de continuer à adopter une posture facilitatrice face aux difficultés d'analyse que semble éprouver le stagiaire.

Parallèlement, l'importance que MS 2 accorde au sens des activités, et notamment au sens que le stagiaire leur confère, la conduit, pendant l'observation de la leçon donnée, à établir un diagnostic de situation en relevant des moments de son activité qui lui semblent avoir dysfonctionné. L'analyse de l'activité de MS 2 montre en effet qu'elle renvoie au stagiaire des moments de la séance qu'elle décrit avec précision : elle le met face à un dysfonctionnement sans le lui présenter comme tel et le questionne à ce sujet 
(intérêt et objectif de la leçon d'arts, respect de la préparation, relation à l'enfant). Le fait de renvoyer le stagiaire à des moments de son activité est caractéristique d'un des gestes définis par Jorro (2016) pour l'accompagnement lié à l'action de «traducteur ». L'auteur précise que ce geste consiste à rendre transparent, autrement dit à mettre devant les yeux un élément qu'il n'aurait pas considéré. Néanmoins, cette pratique peut comporter un risque, si l'objectif poursuivi est la réflexivité du stagiaire. En effet, en choisissant ellemême la porte d'entrée de l'analyse réflexive (Berckers, 2012), l'accompagnatrice risque d'influencer les propos du stagiaire qui suivront. En effet, le fait de pointer la situation peut révéler son point de vue critique, risquant ainsi d'orienter la réponse du stagiaire et d'engendrer chez lui des conduites auto-justificatrices.

Remarquons toutefois que lors du deuxième entretien, la maitre de stage interroge davantage le stagiaire et tente de ne pas se "limiter " à un simple rappel des faits couplé à sa propre analyse de la situation. Elle l'amène à verbaliser à propos du sens qu'il donne à ses activités d'enseignement favorisant alors les attitudes d'investigation et de compréhension (Dugal, 2009).

Liens entre les données du débriefing et celles de l'entretien d'explicitation de MS 2 pour la variable "sentiment de compétence du stagiaire"

Le concept pragmatique concernant le développement professionnel du stagiaire à long terme repose sur une deuxième variable fonctionnelle : « le sentiment de compétence du stagiaire ». Lorsque MS 2 perçoit que le stagiaire évalue négativement ses compétences (indicateur) et qu'il ne s'est pas senti à la hauteur, elle cherche à en comprendre la raison avant de lui livrer sa propre analyse de la situation et de lui expliquer comment il aurait pu s'y prendre pour rendre ses démarches plus efficaces. 
Tableau 6 : Liens entre les données du débriefing et celles de l'entretien d'explicitation de MS 2 pour la variable « sentiment de compétence du stagiaire ».

Table 6: Links between data from the debriefing and those from the clarificatory interview of MS 2 for the functional variable "trainee's perceived competence"

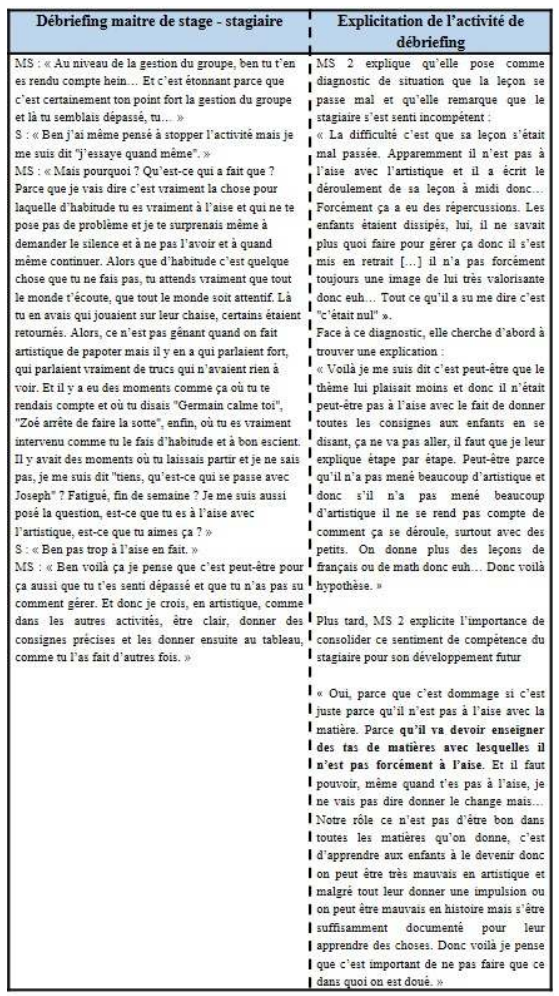

L'analyse de cette variable rejoint le constat établi plus haut: la MS 2 oscille entre les deux postures établies par Schön (1988). Elle semble en effet toujours chercher à favoriser la réflexivité du stagiaire en le questionnant et en le faisant réfléchir à ses pratiques. Cependant la prise en compte d'indicateurs pendant l'observation de la leçon (activité ne se déroulant pas bien) ou pendant le débriefing (sentiment d'incompétence exprimé par le stagiaire) l'amène à agir comme un expert et à resserrer son cadrage.

À nouveau, comme l'indiquent les verbatim issus du débriefing, MS 2 pointe un moment de l'activité ; ici, en l'occurrence, quelque chose qui n'a pas fonctionné ou qui aurait pu être amélioré. Elle questionne le stagiaire à ce sujet et donne son point de vue ainsi que des conseils. L'adoption de cette posture de conseiller peut être caractéristique d'une posture d'expert (Schön, 1988). En effet, l'expert fournit lui-même des solutions. Et c'est bien ici de solutions dont il va s'agir puisque la maitre de stage a identifié un problème : le stagiaire se sent incompétent. MS 2 souligne à plusieurs reprises l'aisance du stagiaire dans la gestion de groupe, ce qui reflète une posture de soutien (Dugal, 2009).

\subsection{Quels liens pouvons-nous entrevoir entre l'activité observée du maitre de stage, la verbalisation par le maitre de stage de son activité et les bénéfices retirés par le stagiaire?}

58 Le témoignage des deux stagiaires laisse entendre que ceux-ci semblent plus satisfaits de l'accompagnement prodigué lorsque leur maitre de stage adopte une posture d'expert. Chez MS 1, la fonction d'expert assurée a permis, selon les dires du stagiaire, de garder un 
sentiment de confiance et de sécurité. Lorsque celui-ci s'exprime sur les bénéfices qu'il retire de ce stage, il mentionne la fonction de soutien assurée par sa maitre de stage qui le mettait en confiance et le rassurait sur sa compétence. Il semblerait donc que le genre « accompagnement - expert » ait satisfait le stagiaire dans le sens où cela lui a permis de reprendre confiance en ses capacités. Peut-être le fait d'avoir été guidé et d'avoir reçu beaucoup de solutions directes l'a-t-il aidé dans cette voie.

Le stagiaire de MS 2 a indiqué s'être amélioré au niveau de la rigueur, de l'organisation et de la remise en question. Le genre professionnel de MS 2 qui oscille entre les postures d'expert et de facilitateur parait avoir permis au stagiaire de s'améliorer dans des domaines importants de son développement professionnel. Cette alternance de postures pourrait lui avoir permis à la fois de s'exercer à la remise en question tout en améliorant ses démarches d'enseignement grâce à des conseils précis.

\subsection{Comparaison des deux maitres de stage}

60 Le sujet ayant la tâche effective la plus éloignée de ses pratiques déclarées est MS 1. En effet, de ses propos, extraits des entretiens d'explicitation ou du premier entretien semidirigé, émerge l'idée qu'elle agit comme un facilitateur qui amènerait son stagiaire à problématiser. L'analyse des deux débriefings indique que dans l'activité, une posture d'expert domine. Cette maitre de stage passe de fait beaucoup de temps à fournir des solutions concrètes au formé, notamment pour ses leçons à venir, ce que l'on peut rapprocher de l'attitude de "décision» (Dugal, 2009). Si le retour sur son action lui a permis de prendre conscience de l'importance de son temps de parole pendant l'entretien, elle n'a rien verbalisé concernant le fait qu'elle n'amenait pas son stagiaire à problématiser et n'a donc pas fait part explicitement d'éventuelle tension identitaire (Bajoit, 2003).

61 Comme indiqué au tableau 1 de la méthodologie, MS 2 se différencie de sa collègue MS 1 par le fait d'avoir suivi une formation de maitre de stage. Contrairement à MS 1, la description qu'elle donne de sa pratique correspond dans une certaine mesure à l'analyse de son activité. Elle a beaucoup verbalisé à propos des notions de sens et de réflexivité du stagiaire, éléments qui semblent bien constituer une des composantes de son modèle opératif. Par ailleurs, elle est consciente de la fonction de conseil qu'elle adopte lorsque la réflexion du stagiaire n'est pas aboutie. Dans sa verbalisation, c'est son côté « facilitateur » qui était le plus mis en avant. Dans l'activité effective, les deux postures se sont concrétisées de façon relativement équilibrée.

62 Enfin, dans les pratiques déclarées des deux sujets, une préoccupation et un sentiment d'agir pour favoriser la réflexion sur son action apparaissent, ce qui indique que pour eux, un «bon» maitre de stage agit comme un facilitateur. Si MS 1 semble plus préoccupée du résultat à atteindre en termes de prise de conscience par le stagiaire des effets de son activité, MS 2 apparait plus guidée par la recherche du développement professionnel futur de son stagiaire (la réflexivité étant une variable fonctionnelle). Au niveau de la tâche réalisée, les fonctionnements lors du débriefing diffèrent. L'une ne parait pas mettre en œuvre la posture déclarée et s'oriente davantage dans une attitude d'expert. L'autre elle oscille entre les deux postures.

63 Enfin, le choix d'un cadre d'analyse de l'activité, basé sur la prise en compte d'indicateurs liés à la situation telle que Pastré (2011) le conçoit, nous conduit à souligner l'importance de la prise en compte des caractéristiques de la situation par chacun des sujets. La nature 
des indicateurs pris par les deux maitres de stage diffère et conduit l'une des deux maitres de stage à réduire l'écart entre tâche redéfinie et tâche effective. Leurs conceptions sur l'accompagnement, au niveau prédicatif, semblent rejoindre la théorie. Sur le plan opératif, en revanche, elles s'en éloignent chacune dans des mesures différentes.

\section{Discussion et perspectives} prescrits. Tâche discrétionnaire de nature, ce rôle implique une "obligation de résultats sans certitude de moyens » (Pastré, 2007, p. 87). Dès lors, notre premier objectif de recherche était de déterminer les moyens mis en œuvre par les deux maitres de stage observées pour assurer leur fonction lors des tâches de débriefing et, surtout, d'identifier les concepts qui organisaient leur activité. Ainsi, en construisant les modèles opératifs de ces deux maitres de stage sur la base des entretiens d'explicitation, nous avons pu mieux comprendre la façon dont leur activité effective différait lors de débriefings postobservation, tant par les concepts pragmatiques mobilisés que par les indicateurs pris dans l'action. En effet, l'activité lors de la tâche de débriefing de MS 1 était principalement guidée par les concepts "prise de conscience du stagiaire " et " décrochage ", tandis que celle de MS 2 était plutôt guidée par un concept général et central que nous avons nommé "développement professionnel du stagiaire». Par ailleurs, les deux maitres de stage se différencient encore au niveau de leur posture: adoption plutôt d'une posture d'expert pour MS 1 et une oscillation entre les postures d' expert et de facilitateur pour MS 2 . Si l'activité effective des deux maitres de stage lors des débriefings et leur genre professionnel diffèrent, elles avaient pourtant toutes deux une représentation assez similaire du rôle qui leur était confié et de la posture qu'elles souhaitaient adopter lors de ces tâches de débriefing : celle du facilitateur (Dugal, 2009). En outre, les entretiens semi-directifs menés à l'étape 1 ont permis de mettre en évidence que cette représentation commune avait pour point de mire le développement professionnel du stagiaire et son autonomie réflexive.

Par ailleurs, les données récoltées lors de l'étape 5 montrent que les deux stagiaires déclarent être pleinement satisfaits de l'accompagnement reçu lors des débriefings, même le stagiaire dont le maitre de stage (MS 1) avait plutôt adopté une posture d'expert. $\mathrm{Au}$ regard de la littérature scientifique qui met en évidence que cette posture d'expert devrait être moins porteuse que la posture de facilitateur (Dugal, 2009; Schön, 1988), ces résultats interpellent. Bien évidemment, les déclarations des stagiaires ne prévalent pas une analyse des réels bénéfices en termes de développement professionnel qui pourrait donner de tout autres résultats. Mais la question subsiste : pourquoi le stagiaire de MS 1 a-il une perception aussi positive de l'accompagnement dispensé par sa maitre de stage lors des tâches de débriefings? Nous formulons deux hypothèses explicatives. La première postule que, dans la perspective où les préoccupations du stagiaire étaient davantage de survie (Füller, 1969), un cadrage plus serré du maitre de stage pourrait profiter - du moins à court terme - au stagiaire, en lui donnant accès à des solutions pratiques, à des « recettes » (Perrenoud, 2013). Ne pas en comprendre les ingrédients pourrait, à ce stade, tout de même conduire le stagiaire à une réussite; grâce à cette recette, il parvient la plupart du temps à gérer le cadre de la classe, et là est tout ce qui l'importe. La seconde hypothèse se rapporte au potentiel manque de prise de conscience 
du stagiaire: s'il ne ressent pas un déséquilibre ou une rupture, nous pouvons faire l'hypothèse qu'il ne ressent peut-être pas le besoin d'être soutenu dans son développement par son maitre de stage. Par ailleurs, le fait que le maitre de stage fournisse conjointement son interprétation de la situation et un conseil d'action pourrait freiner la prise de conscience du stagiaire ou du moins l'adoption d'une posture réflexive (Dugal, 2009).

Trois constats principaux et pistes de prolongement se dégagent de ces résultats. Premièrement, malgré le manque de prescrit, il semble que les maitres de stage considèrent toutes deux qu'une des tâches fondamentales relatives à leur rôle est d'aider leur stagiaire à pouvoir atteindre un niveau de réflexivité plus élevé. Il serait intéressant de voir dans quelle mesure cette redéfinition de la tâche prescrite est partagée par les autres maitres de stage de Fédération Wallonie-Bruxelles. Par ailleurs, une définition du genre professionnel (Clot, 1999; Saujat, 2004) pourrait être réalisée en se basant sur un modèle structuré autour de concepts centraux liés à l'accompagnement et qui pourraient être le reflet de l'aspect personnel de la prise de la fonction. Cette première perspective rejoint les pistes de prolongements envisagées par Filliettaz, Rémery et Trébert (2014, p. 21) qui, dans le domaine de la petite enfance, considèrent que la construction et la visibilité du genre professionnel constituent probablement « un des enjeux majeurs pour la professionnalisation des référentes professionnelles dans ce domaine socio-éducatif spécifique ».

Deuxièmement, entre les intentions d'action de MS 1 et son activité effective, nous pouvons constater qu'il y a un décalage assez important, décalage qui n'est pas conscientisé par la maitre de stage. Ce résultat rejoint le constat formulé par Perrenoud (2013): les formateurs de terrain partagent en grand nombre l'objectif d'aider leurs stagiaires à accéder à davantage de réflexivité sans toutefois être enclins à les y aider pleinement lors de l'activité de débriefing. Réfléchir conduit au doute et le doute conduit à poser des questions qui peuvent mettre à mal le maitre de stage dans sa propre identité professionnelle et l'amener à délaisser la posture de facilitateur au profit de celle d'expert. Selon Perrenoud (2013), le mot d'ordre de ces maitres de stage pourrait ainsi se résumer : « soyez réflexifs, mais, surtout, ne troublez pas l'ordre des choses!»

Troisièmement, dans le cas de MS 1, il semble que le développement professionnel du stagiaire passe parfois en second plan au profit d'un objectif exclusivement ciblé sur les apprentissages des élèves de la classe. Il conviendrait, à ce titre, de mieux prendre en compte la dimension relationnelle qui influence la tâche de débriefing lors de l'accompagnement: que ce soit pour "protéger » ses élèves, le stagiaire ou lui-même, l'enseignant est influencé lors de son activité par ces différents aspects relationnels (Vinatier, 2007). Dans cette perspective, l'identité professionnelle de ces enseignants qui endossent, pour un temps limité, un rôle de formateur constitue une piste de développement professionnelle majeure. Ainsi, outre une orientation méthodologique prenant davantage en considération l'effet des aspects relationnels dans la tâche de débriefing, une autre piste de prolongement consisterait à se pencher sur le modèle opératif de l'activité d'enseignement des maitres de stage lorsqu'ils sont en activité dans leur classe. En effet, les concepts pragmatiques qui guident leur activité pourraient vraisemblablement orienter leur manière d'observer les activités du stagiaire et de les envisager lors du débriefing. En outre, la nature interactionniste de cette tâche de débriefing implique que le maitre de stage n'est pas le seul responsable de son 
déroulement (Vinatier, 2007). Ce dernier élément pourrait expliquer en partie le décalage observé chez MS 1 entre tâche redéfinie et effective.

En guise de conclusion, bien que la fonction d'accompagnement manque de prescrits et qu'elle soit difficilement appréhendable pour les professionnels de terrain, un nombre considérable d'enseignants l'endossent chaque année pour essayer, à leur échelle, de contribuer au développement professionnel d'un futur enseignant. Comme souligné par Rey (2001), cette fonction de maitre de stage gagnerait par conséquent à être davantage formalisée dans les prescrits en FW-B. Gage d'une meilleure compréhension des enjeux de la tâche qui leur est confiée et des moyens possibles pour y parvenir, ce référentiel constituerait une base de réflexion sur laquelle pourraient s'appuyer des dispositifs de formations initiale et continue afin d'aider les maitres de stage à se redéfinir la tâche et de les soutenir dans sa mise en œuvre.

\section{BIBLIOGRAPHIE}

Altet, M., Paquay, P., \& Perrenoud, P. (2002). Formateurs d'enseignants. Quelle professionnalisation? Bruxelles : De Boeck.

Bajoit, G. (2003). Le changement social : approche sociologique des sociétés occidentales contemporaines. Paris : Armand Colin.

Barnier, M. (2001). Le tutorat dans l'enseignement et la formation. Paris : L'Harmattan.

Beauvais, M. (2008). Accompagner, c'est juger ? Éducation permanente, 175, 123-135.

Beckers, J. (2012). Compétences et identité professionnelles : l'enseignement et autres métiers de l'interaction humaine. Bruxelles : De Boeck.

Beckers, J., Biemar, S., Boucenna, S., Charlier, E., François, N., \& Leroy, Ch. (2013). Comment soutenir la démarche réflexive? Outil et grilles d'analyse des pratiques. Bruxelles : De Boeck.

Boru, J.-J. (1996). Du tuteur à la fonction tutorale : contradictions et difficultés de mise en œuvre. Recherche et formation, 22(1), 99-114.

Clot, Y. (1999). La fonction psychologique du travail. Paris : PUF.

De Backer, B. (2004). La formation des accueillantes des milieux d'accueil par le biais du tutorat, étude qualitative. Rapport de recherche pour la Fédération des institutions médico-sociales (FIMS asbl), avec le soutien de la Communauté française. http://www.apefasbl.org

Décret Formation Initiale : décret définissant la formation initiale des instituteurs et des régents. (2001). Moniteur belge, 19/01, p. 1471.

Décret missions : décret définissant les missions prioritaires de l'enseignement fondamental et de l'enseignement secondaire et organisant les structures propres à les atteindre. (1997). Moniteur belge, 23/09, p. 24653.

Dubar, Cl. (2000). La socialisation, construction des identités sociales et professionnelles (3éd.). Paris : A. Colin. 
Dugal, J.-P. (2009). Attitudes dans les entretiens-conseils et formation des conseillers pédagogiques. Travail et formation en éducation [En ligne], 4. https://journals.openedition.org/ tfe/899

Filliettaz, L., Rémery, V., \& Trébert, D. (2014). Relation tutorale et configurations de participation à l'interaction : analyse de l'accompagnement des stagiaires dans le champ de la petite enfance. Activités, 11(1), 22-46. Retrieved from https://journals.openedition.org/activites/288

Forest, L., \& Lamarre, A.-M. (2009). Accompagner des stagiaires vers la maitrise des compétences en enseignement. Québec : Les Éditions de la Chenelière.

Fuller, F. (1969). Concerns of Teachers: a developmental conceptualization. American Educational Research Journal, 6(2), 207-226.

Jorro, A. (2016). Postures professionnelles des conseillers en évolution professionnelle. Revue Internationale de Pédagogie de l'enseignement Supérieur [RIPES], 32(3).

L'Écuyer, R. (1987). L'analyse de contenu : notions et étapes. In J.-P. Deslauriers (Ed.), Les méthodes de la recherche qualitative (p. 49-65). Sillery, QC : Presses de l'Université du Québec.

Leplat, J. (2011). Mélanges ergonomiques : activité, compétence, erreur. Paris : Octarès Éditions.

Maes, O., Colognesi, S., \& Van Nieuwenhoven (2018). « Accompagner/former » ou « évaluer/ vérifier ». Une tension rencontrée par les superviseurs de stage des futurs enseignants? Éducation \& Formation, e-308, 95-106. http://revueeducationformation.be/include/download.php? idRevue $=28 \&$ idRes $=302$

Musquer, A., \& Fabre, M. (2011). Qu'est-ce que conseiller veut dire ? L'entretien conseil comme problématisation « après-coup » de la pratique. In J.-Y. Robin \& I. Vinatier (Eds.), Conseiller et accompagner, un défi pour la formation des enseignants (p. 105-138). Paris : L'Harmattan.

Pastré, P. (2005). Genèse et identité. In P. Rabardel \& P. Pastré (Eds.), Modèles du sujet pour la conception (p. 231-259). Toulouse : Octarès Éditions.

Pastré, P. (2007). Quelques réflexions sur l'organisation de l'activité enseignante. Recherche et Formation, 56, 81-93.

Pastré, P. (2011). La didactique professionnelle : approche anthropologique du développement chez les adultes. Paris : Presses Universitaires de France.

Pastré, P., Mayen, P., \& Vernaud, G. (2006). La didactique professionnelle. Revue française de pédagogie, 154, 145-198.

Perrenoud, P. (2013). La blessure la plus rapprochée du soleil. Ambivalences et résistances face à la posture réflexive. In A. Marguerite, J. Desjardins, E. Richard, L. Paquay, \& P. Perrenoud (Eds.), Former des enseignants réflexifs (p. 79-96). Louvain-la-Neuve, Belgique : de Boeck.

Pirard, F, François, N, \& Noël, S. (2015). Soutien au tutorat et à l'encadrement des stages dans le secteur des milieux d'accueil d'enfants (0-12 ans) : Carnet de bord.

Portelance, L. (2008). L'apport attendu des enseignants associés à la formation des stagiaires. In M. Boutet \& J. Pharand (Eds.). L'accompagnement concerté des stagiaires en enseignement. (p. 53-71). Québec : Presses de l'Université du Québec.

Rey, B. (2001). Recherche sur le rôle des maitres de stage dans la formation initiale des enseignants. Rapport de recherche. Bruxelles, Université Libre de Bruxelles.

Saujat, F. (2004). Comment les enseignants débutants entrent dans le métier ? Formation et pratiques d'enseignement en questions. Revue des HEP de Suisse Romande et du Tessin, 1, 97-106. 
Schön, D. A. (1988). Coaching reflective teaching. In P. P. Grimmett \& G. L. Erickson (Eds.), Reflexion in teacher education (p. 19-29). Vancouver : Pacific educationnal press, University of British Columbia ; New-York ; London : Teacher College Press.

Teissier, R. (1992). Consultation : expertise et facilitation. In R. Teissier \& Y Tellier (Eds.), Méthodes d'intervention, consultation et formation. Sillery. P.Q. : Presses de l'Université de Québec.

Van Nieuwenhoven, C., \& Colognesi, S. (2013). Une recherche collaborative autour des difficultés des maitres de stage à accompagner leur stagiaire. Interacçóes, 27, 118-138.

Van Nieuwenhoven, C., \& Colognesi, S. (2015). Une recherche collaborative sur l'accompagnement des futurs instituteurs : un levier de développement professionnel pour les maitres de stage. Évaluer. Journal international de recherche en éducation et formation, 1(2), 103-121.

Vermersch, P. (1994). L'entretien d'explicitation. Paris : ESF.

Vermersch, P. (2004). Aide à l'explicitation et retour réflexif. Éducation permanente, 160, 71-80.

Veyrac, H. (1998). Approche ergonomique des représentations de la tâche pour l'analyse d'utilisations de consignes dans des situations de travail à risques. Thèse de doctorat en Psychologie, Toulouse : Université Toulouse le Mirail-Toulouse II.

Vidal-Gomel, C., \& Rogalski, J. (2007). La conceptualisation et la place des concepts pragmatiques dans l'activité professionnelle et le développement des compétences. Activités, 4(1), 49-84. https://doi.org/10.4000/activites.1401

Vinatier, I. (2009). Pour une didactique professionnelle de l'enseignement. Rennes : Presses universitaires de Rennes.

Vinatier, I. (2007). La notion d'organisateur dans une perspective interactionniste. Définitions et enjeux. Recherche et Formation, 56, 33-46. http://rechercheformation.revues.org/860

Weill-Fassina, A., Rabardel, P., \& Dubois, D. (Eds.) (1993). Les représentations pour l'action. Toulouse : Octarès.

\section{NOTES}

1. Les tâches de débriefing ont eu lieu après deux temps d'observation différents. Les débriefings de MS 1 et de son stagiaire portaient respectivement sur une leçon de religion (ER1) et une leçon d'écriture (ER2). Les débriefings de MS 2 portaient quant à eux sur une leçon d'éducation artistique (ER1) et sur une leçon de mathématiques (ER2).

\section{RÉSUMÉS}

Cette étude s'ancre dans le champ de la didactique professionnelle (Pastré, Mayen, \& Vergnaud 2006). Elle vise d'une part l'analyse du modèle opératif de maitres de stage impliqués dans la formation initiale des instituteurs primaires et, d'autre part, l'étude des bénéfices retirés de l'accompagnement par leurs stagiaires respectifs. L'article décrit la manière dont deux enseignantes endossent cette fonction dans le contexte de la Fédération Wallonie-Bruxelles, où la 
fonction de maitre de stage manque de prescrits et de clarté dans la définition des tâches qui s'y réfèrent. Le cadre de la didactique professionnelle, qui vise la compréhension de l'activité d'un professionnel en situation, a permis de mettre en évidence des éléments qui guident l'activité des maitres de stage. Par ailleurs, grâce à l'observation de leur activité pendant les débriefings et à la description et l'analyse de ce que les maitres de stage en disent a posteriori lors des entretiens d'explicitation (Vermersch, 1994), des concepts organisateurs de leur action ont pu être établis. Ces résultats sont ensuite confrontés aux données recueillies auprès des stagiaires concernant les bénéfices perçus de l'accompagnement. Si les résultats pointent chez les deux maitres de stage des préoccupations communes ciblées à la fois sur le développement émotionnel et cognitif de leur stagiaire, celles-ci se traduisent toutefois par des postures d'accompagnement différentes. Par ailleurs, des écarts entre le discours des maitres de stage et leur action en situation ont pu être pointés.

This study is anchored in the field of professional didactics (Pastré, Mayen, \& Vergnaud 2006). It aims, on the one hand, to analyze the operative model of two supervisors involved in the initial training of elementary school teachers and, on the other hand, to study the pre-service teachers' perceptions of the benefits of the support provided. This study describes how two teachers take on this supervisory role in the context of French-speaking Belgian education, where neither the supervisory function nor the associated professional tasks are defined. The theoretical framework of the professional didactics, which is based on the reflexive stance that professionals adopt to revisit their activity, allowed me to highlight the conceptual elements that guide the activity of supervising. Moreover, through the observation of their activity during the debriefings with the trainees and through the analysis of what those supervisors said after the debriefing (using clarificatory interview techniques; Vermersch, 1994), a certain number of organizing concepts of their activity have been established. These results were then compared with the data collected from the trainees regarding the perceived benefits of the support received during their placement. The results reveal that the supervisors share concerns about the emotional and cognitive development of their trainee. However, these concerns translated into different postures of support. Furthermore, I identified some gaps between the way these professionals describe their activity and their actual activity.

\section{INDEX}

Keywords : pre-service teacher training, supervisors, support postures, analysis of activity

Mots-clés : formation initiale des enseignants, maitres de stage, postures d'accompagnement, analyse de l'activité

\section{AUTEURS}

\section{CHARLOTTE DEJAEGHER}

ULiège, Faculté de Psychologie, Logopédie et Sciences de l'Éducation, Quartier Agora, Place des orateurs 2 (Bat. B32), 4000 Liège 1, Belgique

cdejaegher@uliege.be

\section{FANNY WATELET}

ULiège, Faculté de Psychologie, Logopédie et Sciences de l'Éducation, Quartier Agora, Place des orateurs 2 (Bat. B32), 4000 Liège 1, Belgique

Fanny.Watelet@alumni.uliege.be 


\section{YVES DEPLUVREZ}

ULiège, Faculté de Psychologie, Logopédie et Sciences de l'Éducation, Quartier Agora, Place des orateurs 2 (Bat. B32), 4000 Liège 1, Belgique yves.depluvrez@uliege.be

\section{STÉPHANIE NOËL}

ULiège, Faculté de Psychologie, Logopédie et Sciences de l'Éducation, Quartier Agora, Place des orateurs 2 (Bat. B32), 4000 Liège 1, Belgique

snoel@uliege.be

\section{PATRICIA SCHILLINGS}

ULiège, Faculté de Psychologie, Logopédie et Sciences de l'Éducation, Quartier Agora, Place des orateurs 2 (Bat. B32), 4000 Liège 1, Belgique

patricia.schillings@uliege.be 